\title{
WHO Follicular Lymphoma Histologic Grade 2
}

National Cancer Institute

\section{Source}

National Cancer Institute. WHO Follicular Lymphoma Histologic Grade 2. NCI Thesaurus.

Code C162999.

6-15 centroblasts per high power field. 